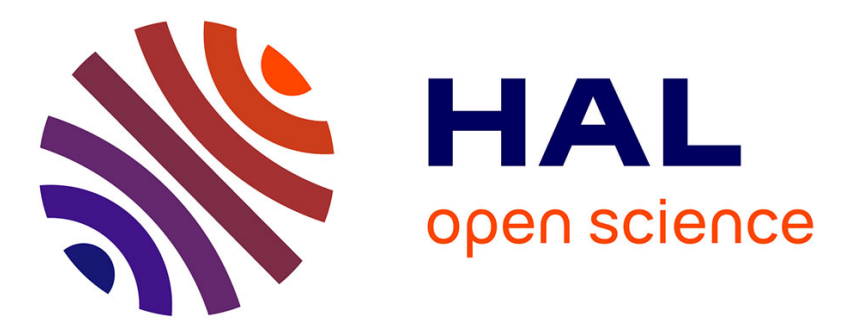

\title{
Karim Taharount, "On est chez nous", Histoire des tentatives d'organisation politique de l'immigration et des quartiers populaires (1981-1988), Solnistata, 2017
} Jeanne Demoulin

\section{- To cite this version:}

Jeanne Demoulin. Karim Taharount, "On est chez nous", Histoire des tentatives d'organisation politique de l'immigration et des quartiers populaires (1981-1988), Solnistata, 2017. Histoire urbaine, 2020, pp.198. 10.3917/rhu.057.0198 . hal-03086112

HAL Id: hal-03086112

https://hal.parisnanterre.fr/hal-03086112

Submitted on 22 Dec 2020

HAL is a multi-disciplinary open access archive for the deposit and dissemination of scientific research documents, whether they are published or not. The documents may come from teaching and research institutions in France or abroad, or from public or private research centers.
L'archive ouverte pluridisciplinaire HAL, est destinée au dépôt et à la diffusion de documents scientifiques de niveau recherche, publiés ou non, émanant des établissements d'enseignement et de recherche français ou étrangers, des laboratoires publics ou privés. 
Karim Taharount, "On est chez nous", Histoire des tentatives d'organisation politique de l'immigration et des quartiers populaires (1981-1988), Solnistata, 2017 (Jeanne Demoulin)

Les quartiers populaires ne sont pas des déserts politiques. C'est ce que nous rappelle l'ouvrage de Karim Taharount qui nous emmène dans un voyage « à hauteur d'homme » ( $\mathrm{p}$. 25) à travers l'histoire de la « mouvance autonome des jeunes immigrés » entre 1981 et 1988. Par le prisme de plusieurs figures militantes que l'on (re)découvre au fil de l'ouvrage et en s'appuyant sur une documentation très riche, l'auteur trace les contours d'une « nébuleuse » (p. 20) et les enjeux et les tensions qui l'ont traversée. Il donne un éclairage précieux sur les «tentatives d'organisation politique » des jeunes immigrés ou issus de l'immigration et permet de saisir la complexité d'un phénomène trop souvent homogénéisé. Dans le même temps, au vu des parallèles qui s'établissent entre hier et aujourd'hui, et de l'histoire récente sur le sujet que rappelle brièvement l'épilogue, l'ouvrage contient nombre de clés pour comprendre la structuration actuelle des jeux d'acteurs et les enjeux qui traversent les organisations.

Le premier objectif du livre est « de comprendre les raisons de [la] stagnation » de la « mouvance» (p. 25), qui n'a jamais dépassé le stade informel malgré les tentatives de structuration à l'échelle nationale. Le deuxième objectif est d'en évaluer les effets à l'aune des attentes de ses membres, objectif plus difficile à atteindre mais pour lequel l'auteur apporte des éléments de réponse.

Le propos s'articule autour d'un « prologue », de deux grandes parties, d'une conclusion et d'un épilogue. Le prologue rappelle les hésitations des jeunes immigrés entre leur pays d'origine et la France, ainsi que les mauvaises conditions de vie et les situations de mise à l'écart que connaissent beaucoup d'entre eux. Il rappelle également les hésitations des différents partis politiques à l'égard des immigrés, et notamment la position ambiguë du Parti communiste. La «mouvance » s'est ainsi constituée autour de sujets en prise directe avec la lutte contre les inégalités. C'est à la lumière de ce contexte que l'on comprend que l'autonomie a constitué un mot de ralliement même si de nombreuses conceptions de ce terme existent et s'affrontent : une « volonté de s'organiser indépendamment du contrôle de l'État et des collectivités locales, mais aussi indépendamment du contrôle exercé par l'ensemble des corps de métiers qu'ils perçoivent comme chargés de leur surveillance ou de leur encadrement » (p. 68), ainsi que vis-à-vis des associations créées par la génération de leurs parents, « trop tournées vers les pays d'origines ».

La première partie met en évidence l'ensemble des conditions et des facteurs du « rapprochement » entre des associations de jeunes immigrés de différentes cités : les luttes pour l'accès au logement (chapitre 1), contre les crimes racistes et sécuritaires (chapitre 2) et les rapprochements liés à la " Marche pour l'égalité » (chapitre 3). Karim Taharount prend le parti d'incarner chacun de ces « rapprochements » et retrace le parcours de militants mobilisés sur ces sujets. Il tient ainsi son objectif d'une histoire " à hauteur d'homme », qui donne la parole à des acteurs laissés dans l'ombre de l'histoire légitime. Si la succession de portraits peut perdre le lecteur, plusieurs figures marquantes restent en mémoire. Parmi elles, celles de Norredine Iznasni et des frères Abdallah, militants nanterriens, piliers de l'Association Gutenberg qui se structure autour de la lutte pour le relogement des habitants de la cité de transit du même nom. Ils ont été des acteurs centraux de la Coordination des cités de transit, de l'organisation des Forums justice et de la structuration de l'Association des familles de victimes de crimes racistes et sécuritaires. L'auteur souligne également la place importante de 
femmes, notamment au sein du collectif Zaâma d'Banlieue dans la région lyonnaise. Des collectifs et associations locales se sont ainsi progressivement structurés puis rapprochés à la faveur de revendications communes, au niveau régional mais également national jusqu'à former des réseaux d'échanges informels autour de problématiques communes. Certains rapprochements ont donné lieu à des structurations plus formalisées comme la création de l'agence de presse IM'média. L'auteur revient longuement sur la Marche pour l'égalité et les tensions qui ont marqué son organisation. On peut voir combien cette Marche a autant contribué à rapprocher des collectifs et associations de jeunes immigrés qu'à dessiner voire à creuser des lignes de fracture qui diviseront progressivement mais profondément la "mouvance ". L'association Gutenberg et Zaâma d'Banlieue refusent ainsi de se rallier au projet qui ne relève pas selon elles d'une dynamique autonome et qui interfère ce faisant avec la dynamique qu'elles essayent de mettre en place avec la Coordination des cités de transit.

La deuxième partie met en évidence les tentatives de structuration de la mouvance (chapitre 4) avant de revenir sur les facteurs permettant d'expliquer leur échec (chapitre 5) pour terminer par ses " reflux » (chapitre 6). En donnant là aussi à voir plusieurs figures militantes, parmi lesquelles la désormais célèbre Farida Belghoul, Karim Taharount montre que si les collectifs et coordinations se multiplient à travers la France (Collectif jeunes, Coordination Justice, Collectif Rhône-Alpes, Collectif PACA, Collectif Lille-Roubaix), les divergences ne permettent pas l'avènement d'un "mouvement». Les débats autour des différences de statut social des militants (les moins aisés accusant les autres de ne pas connaître la réalité quotidienne des jeunes immigrés), les tensions relatives au genre (l'engagement dans l'espace public étant mal vu pour les femmes), les différends entre ceux qui ont soutenu la marche et ceux qui l'ont critiquée, les questionnements autour des personnes ou collectifs effectivement légitimes pour prendre part au mouvement (les intellectuels, les organisations politiques, les associations anti-racistes, les jeunes français, les jeunes immigrés non maghrébins peuvent-ils être membres ?) mais également les désaccords autour des termes du programme à adopter (rester sur un discours très général ou formuler des revendications précises relativement aux inégalités et aux discriminations ?) constituent autant de lignes de clivages qui ne trouvent pas d'issue collective satisfaisante. Les débats sont très longs, et, sans surprise, comme on le constate dans d'autres organisations militantes d'hier et d'aujourd'hui, la tension entre efficacité et démocratie est structurante. L'auteur montre ainsi que si une charte de revendications commune est finalement élaborée au cours de l'année 1984, elle n'a jamais été reprise et défendue de manière collective. Les associations et collectifs se sont progressivement repliés sur leurs activités locales. L'échec de «Convergence 84 » et l'avènement d'organisations puissantes " téléguidées par le Parti socialiste » auront finalement raison des tentatives d'organisation d'une mouvance autonome des jeunes immigrés.

L'ouvrage de Karim Taharount propose ainsi une synthèse intéressante sur une histoire méconnue alors même que les enjeux socio-politiques qu'elle recouvre continuent de structurer nombre de problématiques contemporaines. Les inégalités et les discriminations envers les "jeunes immigrés ", leurs parents et leurs enfants mobilisent encore nombre de militants à travers les quartiers populaires qui peinent à obtenir, en dépit de leurs mobilisations, une reconnaissance et des réponses satisfaisantes de la part des pouvoirs publics qui aboutirait à une véritable « égalité de droit et de traitement» (p. 301). 